\title{
Trabajo en equipo en fuentes tradicionales y el desempeño laboral en el Centro Comercial Alameda del Calzado de Trujillo, 2014
}

\author{
Teamwork in traditional sources and job performance in alameda del calzado \\ Trujillo’s mall, 2014 \\ Lorenzo Valderrama Plasencia ${ }^{1}$
}

\section{RESUMEN}

La investigación se basa en «hallazgos» que demuestran la práctica muy ancestral del trabajo en equipo, recurriendo para ello a la información de las fuentes tradicionales sobre las antiguas civilizaciones en el Perú: el Imperio Incaico con las ruinas de Machu Picchu y la Cultura Moche. Tal información fue contrastada mediante las entrevistas a ingenieros y arquitectos, así como mediante una encuesta aplicada a los miembros socios de la «Alameda del Calzado» y permitió conocer y justificar el valor del trabajo en equipos. La muestra es la población conformada por los cien miembros socios. Esta investigación aplicada y descriptiva, además de documental e histórica ha permitido concluir que el trabajo en equipo desde milenios contribuye a la mejora del desempeño laboral cumpliendo el objetivo en el mismo sentido, en especial en el Centro Comercial Alameda del Calzado.

Palabras clave: trabajo en equipo; fuentes tradicionales; desempeño laboral.

\begin{abstract}
The research is based on «findings» that demonstrate the very ancient practice of teamwork, resorting to information from traditional sources about ancient civilizations in Peru: the Inca Empire with the ruins of Machu Picchu and the Moche Culture. Such information was contrasted by interviews with engineers and architects, as well as through a survey of members of the «Alameda del Calzado» members allowed to know and justify the value of teamwork. The sample is the population made up of one hundred associate members in this applied and descriptive research as well as documentary and historical and allowed to conclude that teamwork millennia contributes to improved job performance on target in the same direction especially Footwear in Alameda Mall.
\end{abstract}

Keywords: teamwork; traditional sources; job performance.

1 Universidad Nacional «Santiago Antúnez de Mayolo». Huaraz, Perú. 


\section{ICHIKLLACHAW}

Kay yachakuy ashiy patashqa unay awiluntsikkunapa yanapanakuy uryachawmi, tsaypaqmi ashikashqa unay Piruu awilutsikkunapa yachayninta: Inkakunapa impiriyun, Machu Picchuwan muchik nunakuna. Tsay willakuykunam tinkutsishqa kashqa inhiniirukuna, arquitektukuna uryachashqawan, hina «Alameda del Calzaduchaw» sosyukunatawan, tsaychawmi chanintsaatsikashqa yanapanakuy uryata. Tsaychaw tapushqa kayashqa pachak sosyukuna, kay yachay ashiy kashqa aplicada -descripitivam, hina qillashqachaw ashiy, unay willakuykuna; tsaykunawanmi paqtatsishqa kashqa: ima uryapis allaapam alliyan yanapanakuy uryawan, tsaynawpam tsay Chawpi Rantikuna Alameda del Calzado alli uryan.

Pushaq shimikuna: yanapanakuy urya; unaypita imapis winatsiqkuna; uryachaw imaypis alli ruray.

\section{INTRODUCCIÓN}

El trabajo es la actividad realizada por el hombre que demanda esfuerzo físico y mental con el afán de lograr una determinada meta. El equipo es un conjunto de personas organizadas con un fin común. Respecto al trabajo en equipo, Newstrom (2011: 331) afirma que «cuando los miembros de un equipo de tareas conocen sus objetivos, participan de manera responsable y entusiasta en ellas y se apoyan entre sí, realizan un trabajo en equipo».

Así mismo Whetten y Cameron (2011: 494) manifiestan que «los equipos son grupos de individuos que realizan actividades interdependientes, cuyos comportamientos se ven influidos mediante la interacción. Muchas empresas han atribuido sus mejoras en el desempeño directamente a la conformación de equipos en el lugar de trabajo».

A través de la presente investigación se planteó como objetivo general: Determinar que el trabajo en equipo referido en fuentes tradicionales desde milenios contribuye afirmando la mejora del desempeño laboral en la Alameda del Calzado. Una herramienta de ayuda ha sido una encuesta. Así también definir cómo el trabajo en equipo según fuentes tradicionales sigue vigente; se realizó entrevistas a ingenieros y arquitectos.

En todo equipo de trabajo existen aspectos básicos que el líder debe tomar en consideración. En ese sentido, Urcola (2010) hace hincapié en el hecho de compartir un objetivo común, practicar liderazgo, lograr la cooperación, desarrollar la comunicación y propiciar la participación. Así mismo, Chiavenato (2002) manifiesta que para evaluar el desempeño laboral se cuenta con ciertos elementos tales como conocimiento técnico, logro de metas, trabajo en equipo, comunicación y asesoría. De igual modo, Alles (2013) indica que el análisis del desempeño de una persona es un instrumento para dirigir y supervisar el personal.

Al líder le corresponde desarrollar funciones diversas para maximizar las potencialidades del equipo. En ese contexto, Urcola (2010) subraya que se debe señalar claramente los objetivos entre los miembros de la organización, así también que la comunicación debe ser clara. De otro lado, también señala que frente a las tareas difíciles debe solicitarse colaboración, evitar enfrentamientos, asignar tareas, entre otras actividades. 
Por otro lado, se puede señalar que a medida que la humanidad avanza va marcando sucesos y eventos que con el transcurrir del tiempo se convierten en historia, a ellos se les llama fuentes históricas o tradicionales. Bajo ese escenario se acudió a la información de las fuentes tradicionales de diferentes naciones que asombraron al mundo con sorprendentes hallazgos físicos. Aunque es preciso aclarar que para ese tiempo no existía aún como tal la doctrina del trabajo en equipo, para su justificación se practicó entrevistas a ingenieros y arquitectos.

Según Kauffmann (2011) una obra maravillosa y que sigue asombrando al mundo entero son las ruinas de Machu Picchu, (del quechua sureño machu pikchu, «Montaña Vieja»), situados en Cuzco, Perú. La superficie edificada es aproximadamente de 530 metros de largo por 200 de ancho y cuenta con 172 edificios en su área urbana. Es probable que su construcción -hecha por etapas- haya tomado alrededor de 20 años.

Así mismo, Leveratto (2010), manifiesta que otra gran obra constituye la fortaleza de Sacsayhuamán, es decir, «lugar donde se sacia el halcón». Su principal característica es que fue construida con grandes bloques de piedra, alcanzando los más altos los $9 \mathrm{~m}$. Se asevera que su construcción demoró 50 años aproximadamente, iniciándose en el período del Inca Túpac Yupanqui. Fue construida por 20.000 hombres.

De igual modo, Bernat (2002) manifiesta que Moche data entre 200 años a.C. y 700 años d.C. y es una de las culturas más conocidas y admiradas del Perú desde el punto de vista de su expresión artística, y se desarrolló en la larga y angosta franja desértica de la Costa Norte del Perú. También se la denomina cultura Mochica en razón del nombre de la lengua, el muchik, que hablaban sus pobladores y ocuparon los valles de Piura, La Leche, Lambayeque, Zaña, Jequetepeque, Chicama, Moche, Virú, Chao, Santa y Nepeña. Los centros principales en Moche Norte fueron el valle del río Jequetepeque (donde se halla San José de Moro y Huaca Dos Cabezas) y el valle del río Lambayeque (donde se halla Sipán y Pampa Grande). Los centros principales de Moche Sur fueron el valle del río Moche (donde se halla la Huaca del Sol y de la Luna) y el valle del río Chicama (donde se halla el Complejo El Brujo).

La base de su desarrollo económico, en un territorio desértico, se fundamentó en una agricultura que producía excedentes gracias al establecimiento de irrigación artificial con acueductos que desviaban hacia acequias el agua proveniente de los ríos que bajan de los Andes y paralelamente desarrollaron habilidades en la pesca. La cerámica Moche, dada la calidad estética y temática de su innumerable producción se ha convertido en el mejor espejo de su vida cotidiana y espiritual. El sitio de Cerro Mayal, que se encuentra en la parte baja del valle de Chicama, ha proporcionado informaciones valiosas para la reconstrucción de las actividades que se realizaban en un gran taller especializado de cerámica; identificándose la variación de los productos manufacturados y reconstruyéndose así la naturaleza tecnológica de fabricación, como el uso de moldes y hornos.

Queda claro que el presente estudio se justifica porque intenta determinar que el trabajo en equipo a través de la historia ha permitido tener los avances y beneficios que hoy en día vemos en nuestra sociedad. En tal sentido, los vestigios físicos como los monumentos arquitectónicos mencionados anteriormente, son considerados actual- 
mente maravillas del mundo porque atraen a muchos turistas. El turismo es actualmente fuente importante de ingreso de divisas, genera mano de obra y origina la aparición de otros negocios conexos que requieren una serie de recursos humanos, financieros, de infraestructura, etc., creándose un gran dinamismo económico que beneficia a la sociedad.

Cabe destacar la importancia del uso de la disciplina del trabajo en equipo en muchos sectores productivos del país, entre los cuales podemos mencionar: cultivos, cría y cuidado de ganado, pesca, extracción de recursos forestales, las maquinarias, las materias primas, la producción de papel y cartón, construcciones, comercio minorista y mayorista, actividades bancarias, asistencia de salud, educación y cultura, etc. En tal sentido el sector del calzado en Trujillo produce y abastece a todo el Perú, siendo el Centro Comercial Alameda del Calzado de Trujillo uno de sus principales ejes de distribución. Hasta este lugar se dan cita personas de todo el país generándose una serie de servicios como financieros, de transportes, hospedajes, alimentación, diversión, turismo, etc. En todo este conglomerado de actividades debe primar un servicio de calidad y es ahí donde conviene destacar el papel importante del trabajo en equipo como una herramienta básica de gestión para mejorar el desempeño laboral y su consecuente beneficio para la comunidad y específicamente en el Centro Comercial Alameda del Calzado.

\section{MATERIALES Y MÉTODOS}

Por tratarse de una población relativamente pequeña y para no afectar la validez de los resultados, la muestra será equivalente a la población, es decir 100 miembros socios de la Alameda del Calzado. Se trata de una investigación aplicada toda vez que parte de una situación problemática que requiere ser intervenida y mejorada en provecho de la sociedad. Los métodos utilizados fueron el hipotético-deductivo, hermenéutico y descriptivo; la técnica empleada fue la encuesta, cuya herramienta es el cuestionario y se ha aplicado al personal de ventas de las tiendas en la Alameda del Calzado. También se ha utilizado la técnica de la entrevista y así mismo se ha empleado la técnica de revisión bibliográfica, debido a que forma parte integral de toda investigación; el diseño de investigación es no experimental, en la modalidad descriptiva transaccional.

\section{RESULTADOS}

La recolección de datos e información obtenida a partir de las entrevistas y encuestas, generó una importante información determinante para la investigación, que en una parte se plasmó para las entrevistas mediante las opiniones vertidas por profesionales especialistas, de entre las cuales se seleccionaron aquellas que respondían directamente al objetivo de la investigación. Por otro lado, para las encuestas se ha formulado un conjunto de preguntas, las cuales fueron seleccionadas, diez de ellas estrictamente también según el objetivo de la investigación; y a su vez estas fueron reagrupadas en bloques de cinco de acuerdo a la variable independiente: trabajo en equipo, y a la variable dependiente: mejora en el desempeño laboral. 
En tal sentido, se muestra los siguientes resultados alcanzados:

\section{a) Entrevista a Arquitectos e Ingenieros:}

Opine Ud. si los líderes de las antiguas civilizaciones para llevar a cabo las asombrosas y complejas construcciones tales como las ruinas de Machu Picchu, la fortaleza de Sacsayhuamán y en la cultura Moche, apelaron al trabajo en equipo:

$\mathrm{R}_{1}$. Al tomar conocimiento de cómo fueron construidas y lo complicado que fue todo ese trabajo, considero que hubo planificación. Opino también que en todo grupo de personas que se reúnen bajo cualquier fin, consideran siempre importante saber comunicar qué es lo que se va a realizar. En un momento determinado se puede pedir el apoyo y la entrega de sus trabajadores y es bueno que los jefes asignen tareas y responsabilidades para evitar enfrentamientos.

$\mathrm{R}_{2}$. En principio, no me queda ninguna duda que hubo planes para edificar las construcciones milenarias. Considero que los jefes serían muy bien preparados y que sabrían llegar a los trabajadores comunicándoles claramente lo que deberían hacer. En cierto momento debieron solicitar de los trabajadores mucha entrega y ganas de hacer las cosas y no descarto la posibilidad que se originaron muchos roces y enfrentamientos que seguro debieron ser controlados por los jefes, además debieron haberles alcanzado sus tareas y responsabilidades.

$\mathrm{R}_{3}$. Considero que hubo la planeación antes de iniciar las construcciones y se entiende que en esta clase de grandes proyectos, se debe recurrir a la colaboración de los operarios; no obstante puedo imaginar que se presentaron muchos problemas, sin embargo el supervisor tendría que afrontar y evitar que se agranden. Considero que el trabajo obedecía al conocimiento de las tareas que cada trabajador tenía de su puesto.

$\mathrm{R}_{4}$. Antes de iniciar estas construcciones creo que se planificaron bien los objetivos mediante la comunicación que es importante en estos casos. Se les debió pedir que se muestren atentos a lo que hagan y seguramente hubo enfrentamientos y que el líder debió asumir la solución. También creo que cada cual sabía sus tareas y responsabilidades.

b) Resultados de la encuesta de trabajo en equipo y desempeño laboral en la «Alameda del Caliado»:

1. Trabajo en equipo

Tabla 1. Indicar si en la empresa se trabaja en función de un objetivo común

\begin{tabular}{lcc}
\hline \multicolumn{1}{c}{ Factores } & $\mathrm{N}^{\mathrm{o}}$ de encuestados & $\%$ \\
\hline Siempre & 70 & 70 \\
Casi siempre & 15 & 15 \\
Indiferente & 00 & 00 \\
Casi nunca & 10 & 10 \\
Nunca & 05 & 05 \\
TOTAL & 100 & 100 \\
\hline
\end{tabular}


En la tabla 1 se puede observar que el 70\% de los trabajadores conoce qué es lo que se espera de ellos y se esfuerzan sin egoísmo por compartir unánimemente sus habilidades que redundarán en satisfacer a los clientes y en el beneficio de los que conforman este consorcio del calzado.

Tabla 2. Existe alguien que siempre se interesa por solucionar los problemas

\begin{tabular}{lcc}
\multicolumn{1}{c}{ Factores } & N $^{\circ}$ de encuestados & $\%$ \\
\hline SI & 83 & 83 \\
NO & 17 & 17 \\
TOTAL & 100 & 100 \\
\hline
\end{tabular}

En la tabla 2 se observa mayoritariamente al 83\% de trabajadores reconociendo la importancia de liderazgo en el desarrollo de sus labores que les permitirá en definitiva brindar un servicio de calidad.

Tabla 3. Dar y recibir apoyo de los compañeros de trabajo

\begin{tabular}{lcc}
\hline \multicolumn{1}{c}{ Factores } & $\mathrm{N}^{\mathrm{o}}$ de encuestados & $\%$ \\
\hline Siempre & 79 & 79 \\
Casi siempre & 03 & 03 \\
Indiferente & 00 & 00 \\
Casi nunca & 06 & 06 \\
Nunca & 12 & 12 \\
TOTAL & 100 & 100 \\
\hline
\end{tabular}

En la tabla 3 se muestra una fuerte aprobación del 79\% en el sentido que su trabajo debe fundamentarse en función de la cooperación e interdependencia frente a los obstáculos que a diario tienen que enfrentar para lograr ser exitosos en sus negocios.

Tabla 4. Se comunican y se discuten alturadamente los posibles desacuerdos

\begin{tabular}{lcc}
\hline \multicolumn{1}{c}{ Factores } & N $^{\mathrm{d}}$ de encuestados & $\%$ \\
\hline Siempre & 83 & 83 \\
Casi siempre & 00 & 00 \\
Indiferente & 00 & 00 \\
Casi nunca & 13 & 13 \\
Nunca & 04 & 04 \\
TOTAL & 100 & 100 \\
\hline
\end{tabular}

En la tabla 4 se observa que el 83\% de trabajadores considera de mucha utilidad la comunicación entre ellos mismos y también cuando tienen que asesorar a sus clientes en relación a los modelos, precios y calidad del calzado que ofertan. 
Tabla 5. Si se toma en cuenta la formulación de las metas

\begin{tabular}{lcc}
\hline \multicolumn{1}{c}{ Factores } & $\mathrm{N}^{\mathrm{o}}$ de encuestados & $\%$ \\
\hline Siempre & 72 & 72 \\
Casi siempre & 06 & 06 \\
Indiferente & 00 & 00 \\
Casi nunca & 14 & 14 \\
Nunca & 08 & 08 \\
TOTAL & 100 & 100 \\
\hline
\end{tabular}

La tabla 5 indica que un $72 \%$ reconoce que son tomados en cuenta mediante sus ideas para la elaboración de los objetivos que como negocio deben alcanzar. Esto es importante porque se sentirán responsables de alcanzar las metas que ellos mismos ayudaron a planear.

\section{El desempeño laboral}

Tabla 6. Poseer cierto grado de experiencia y destreza para el cumplimento de las tareas

\begin{tabular}{lcc}
\hline \multicolumn{1}{c}{ Factores } & N $^{\circ}$ de encuestados & $\%$ \\
\hline Excepcional & 00 & 00 \\
Sobre lo esperado & 03 & 03 \\
Dentro de lo esperado & 79 & 79 \\
Por debajo de lo esperado & 18 & 18 \\
Muy por debajo de lo esperado & 00 & 00 \\
TOTAL & 100 & 100 \\
\hline
\end{tabular}

En la tabla 6 se observa que el 79\% responde que poseen conocimientos técnicos adecuados y por lo tanto están preparados para asesorar a los clientes bajo cualquier circunstancia. De esa manera también aseguran la fidelización de los compradores.

Tabla 7. Cumplimiento de las metas y objetivos trazados

\begin{tabular}{lcc}
\hline \multicolumn{1}{c}{ Factores } & $\mathrm{N}^{\circ}$ de encuestados & $\%$ \\
\hline Excepcional & 00 & 00 \\
Sobre lo esperado & 06 & 06 \\
Dentro de lo esperado & 87 & 87 \\
Por debajo de lo esperado & 07 & 07 \\
Muy por debajo de lo esperado & 00 & 00 \\
TOTAL & 100 & 100 \\
\hline
\end{tabular}

En la tabla 7 se aprecia que un $87 \%$ están seguros y, con su esfuerzo y dedicación en las labores encomendadas, están contribuyendo para que se logre los objetivos planteados. 
Tabla 8. En la empresa se desarrollan las tareas mediante equipos de trabajo

\begin{tabular}{lcc}
\hline \multicolumn{1}{c}{ Factores } & $\mathrm{N}^{\mathrm{o}}$ de encuestados & $\%$ \\
\hline Excepcional & 00 & 00 \\
Sobre lo esperado & 19 & 19 \\
Dentro de lo esperado & 78 & 78 \\
Por debajo de lo esperado & 03 & 03 \\
Muy por debajo de lo esperado & 00 & 00 \\
TOTAL & 100 & 100 \\
\hline
\end{tabular}

En la tabla 8 se indica que el 78\% está convencido que el ambiente laboral en el que se desarrollan es el resultado de trabajar en equipo y que ello está contribuyendo a la mejora del desempeño de sus tareas para brindar un servicio adecuado a sus clientes.

Tabla 9. En sus relaciones laborales y con sus clientes se comunica sin dificultad

\begin{tabular}{lcc}
\hline \multicolumn{1}{c}{ Factores } & $\mathrm{N}^{\mathrm{o}}$ de encuestados & $\%$ \\
\hline Excepcional & 00 & 00 \\
Sobre lo esperado & 54 & 54 \\
Dentro de lo esperado & 33 & 33 \\
Por debajo de lo esperado & 13 & 13 \\
Muy por debajo de lo esperado & 00 & 00 \\
TOTAL & 100 & 100 \\
\hline
\end{tabular}

En la tabla 9 se aprecia que el 54\% valora la comunicación como un factor relevante que les permite actuar con fluidez en su interrelación con los clientes y compañeros de trabajo. Esta actitud se reflejará en mayores ventas y mejoras para los trabajadores.

Tabla 10. Capacidad de brindar asesoría a los clientes y compañeros de trabajo

\begin{tabular}{lcc}
\hline \multicolumn{1}{c}{ Factores } & N $^{\circ}$ de encuestados & $\%$ \\
\hline Excepcional & 00 & 00 \\
Sobre lo esperado & 27 & 27 \\
Dentro de lo esperado & 58 & 58 \\
Por debajo de lo esperado & 15 & 15 \\
Muy por debajo de lo esperado & 00 & 00 \\
TOTAL & 100 & 100 \\
\hline
\end{tabular}

En la tabla 10 se observa que el 58\% considera fundamental brindar asesoría a los compañeros de trabajo y a los clientes en los temas sobre calidad, moda, precio y comodidad del calzado. Esto permitirá una relación muy personalizada con los consumidores quienes sin duda comentarán a otros de sus experiencias vividas, quienes llegarán a este centro comercial en busca de calzado. 


\section{DISCUSIÓN}

De acuerdo a la información obtenida en los resultados de la investigación, se evalúa las opiniones vertidas por los especialistas que corrobora con los autores de las referencias utilizadas sobre las variables de investigación, y se explica del modo siguiente:

a) Entrevista a arquitectos e ingenieros para responder al objetivo: «Definir cómo el trabajo en equipo segun fuentes tradicionales se mantiene vigente»:

Cabe mencionar que para lograr este objetivo se utilizó las fuentes tradicionales para que los especialistas opinen si los líderes de las antiguas civilizaciones para llevar a cabo las asombrosas y complejas construcciones tales como los restos arqueológicos de Machu Picchu, la fortaleza de Sacsayhuamán y en la cultura Moche, apelaron al trabajo en equipo.

En sus opiniones recalcan la importancia de formular objetivos para tener una base a seguir en el trabajo y que esto se logra mediante la aplicación de una correcta y precisa comunicación entre los trabajadores, haciendo que se involucren en sus responsabilidades. También resaltan que las tareas mientras más complejas se vuelven es cuando el líder debe apelar a la entrega e identificación de parte de los trabajadores, haciéndoles sentir necesarios en la ejecución de los proyectos. Así mismo dejan claro que debe gestionarse correctamente los recursos humanos para evitar enfrentamientos, los mismos que retrasan el avance de las obras y que es conveniente que se asigne claramente las tareas y responsabilidades. Todo ello coincide con Urcola (2010), quien señala que el líder de equipo desarrolla funciones que le permitan armonizar en la gestión de los recursos humanos y para ello establece que deben existir objetivos bien definidos mediante la comunicación precisa. También indica que el líder debe solicitar la colaboración cuando la situación lo exige y generar un agradable clima laboral procurando evitar los enfrentamientos. De igual forma manifiesta que debe tenerse presente la correcta asignación de tareas y responsabilidades para evitar duplicidad de funciones. Todo esto confirma que el trabajo en equipo desde milenios se ha venido aplicando en la gestión de recursos humanos y que éste se mantiene vigente.

\section{b) De la encuesta de trabajo en equipo y desempeño laboral en la «Alameda del Calzado»:}

Es preciso mencionar que este objetivo se logró mediante la encuesta a los colaboradores de la Alameda del Calzado de Trujillo y cuya discusión se muestra a continuación:

La tabla 1 muestra que el 70\% considera que siempre se comparte el objetivo, lo cual es fundamental para los trabajadores de la Alameda porque les permite conocer cómo van en sus pretensiones de alcanzar sus metas, mientras que Urcola (2010) indica que en los equipos se debe compartir el objetivo común.

La tabla 2 refiere que el 83\% considera que en su trabajo sí existe liderazgo, que es indispensable que exista una persona que constantemente se esté interesando por los trabajadores dándoles la debida tranquilidad y seguridad, en tanto que Urcola (2010) 
manifiesta que los equipos deben tener un líder.

La tabla 3 indica que el 79\% siempre considera que existe cooperación y este factor resulta vital en los trabajadores, porque les permite experimentar el sentido de unidad y fortaleza frente a los problemas cotidianos que deben afrontar, mientras que Urcola (2010) refiere que la cooperación es fundamental en todo equipo. La tabla 4 muestra que el 83\% de colaboradores siempre cree que no tiene dificultad para comunicarse y esto es digno de resaltar como una ventaja competitiva para resolver las inquietudes de sus clientes y brindarles un servicio de calidad. Al respecto Urcola (2010) puntualiza que los equipos deben operar en un ambiente de comunicación y relación fluida.

La tabla 5 refiere que participa en la formulación de las metas siempre un $72 \%$ y con ello los trabajadores se sienten motivados y comprometidos a esforzarse por conseguir las metas trazadas; sobre ello Urcola (2010) señala que en el trabajo en equipo el líder debe propiciar la participación de los colaboradores en la formulación y ejecución de los objetivos.

La tabla 6 indica que el 79\% considera que posee conocimiento técnico dentro de lo esperado, este resultado es importante porque demuestra que los trabajadores se encuentran permanentemente capacitados y dispuestos a resolver las inquietudes de los clientes y a responder frente a las expectativas de sus jefes, mientras que Chiavenato (2002) señala que para un buen desempeño laboral, el colaborador debe poseer un grado de experiencia para el desarrollo de sus tareas.

En la tabla 7 se observa que un $87 \%$ cree que se logra las metas dentro de lo esperado; esto es una virtud que consolida el esfuerzo y la dedicación de los trabajadores de la Alameda y saben que los beneficiados serán tanto los clientes como ellos mismos; al respecto Chiavenato (2002) indica que los trabajadores con su desempeño colaboran con el logro de las metas.

En la tabla 8 se aprecia que un $78 \%$ cree que el trabajo en equipo se practica dentro de lo esperado y ello es el resultado de todo un mancomunado esfuerzo que muestran cada día los trabajadores de la Alameda, siendo conscientes que no deben descuidarse de ningún detalle, toda vez que la competencia no descansa; al respecto Chiavenato (2002) manifiesta que para un buen desempeño, el colaborador debe saber interrelacionarse e intercambiar conocimientos con los demás.

La tabla 9 refiere que la comunicación con clientes y compañeros está en un 87\% sobre lo esperado y ello es saludable porque permite que se interactúe en un ambiente agradable de trabajo, donde todos saben comunicarse respecto a qué es lo que deben hacer para mantener satisfechos a sus clientes, mientras que Chiavenato (2002) indica que para un buen desempeño el colaborador debe tener la habilidad para escuchar y hacerse escuchar.

En la tabla 10 se observa que los encuestados creen que realizan labor de asesoría so- 
bre lo esperado y dentro de lo esperado en un $85 \%$, lo que contribuye en la confianza y seguridad que debe brindársele a los clientes cuando llegan a la Alameda en busca de calzado y sobre ello Chiavenato (2002) manifiesta que para un buen desempeño, los colaboradores deben tener capacidad de asesoría mostrando conocimiento, lealtad y compromiso.

\section{CONCLUSIONES}

Las entrevistas realizadas a arquitectos e ingenieros confirman que el trabajo en equipo según fuentes tradicionales se mantiene vigente y contribuye a la mejora del desempeño laboral de las actuales organizaciones.

Las encuestas realizadas a los miembros socios de la Alameda del Calzado confirman que el trabajo en equipo influye en la mejora del desempeño laboral. Los efectos favorables que se obtienen son que la Alameda del Calzado es un gran centro comercial que genera un importante número de puestos de trabajo, moviliza una serie de recursos y atrae a compradores de todo el país. Esto conlleva a la necesidad de satisfacer otras exigencias como hospedaje, transporte, alimentación, distracción, generándose un flujo económico muy importante con beneficios para la comunidad.

También es sabido que para la fabricación de calzado se utiliza una serie de insumos en la cual participan diversas empresas como curtiembres, industrias de químicos, fábricas de maquinarias y equipos, empresas de servicios múltiples. En todas ellas se requiere diversos tipos de mano de obra y otros recursos.

Es importante mencionar que la infraestructura actual del Centro Comercial Alameda del Calzado es el fruto del trabajo en equipo de los miembros socios y fabricantes de calzado de Trujillo. Ellos con mucho esfuerzo, desprendimiento y unidad de espíritu decidieron un día crear este emporio comercial que ahora forma parte del importante eje económico de la ciudad de Trujillo.

\section{AGRADECIMIENTOS}

A las autoridades de la Universidad Nacional «Santiago Antúnez de Mayolo», por permitir la consolidación del presente trabajo de investigación.

\section{REFERENCIAS BIBLIOGRÁFICAS}

Alles, Martha. 2013. Construyendo talento. Buenos Aires: Granica.

Bernat, Gabriel. 2002. Cultura Moche. <http://www.gabrielbernat.es/peru/preinca/ cultpreincaicas/dregionales/MOCHE/moche.html> [Consulta: 04-09-2015].

Chiavenato, Idalberto. 2002. Gestión del Talento Humano. Mexico: Mc Graw Hill. 
Kauffmann, Federico. 2011. <Historia de Machu Picchu>. Revista Machupichu 360, No 1, Lima. <http://www.machupicchu360.org/machu-picchu/9/losespecialistas /26/machu-picchu-historia/\&pag=1> [Consulta: 25-08-2015].

Leveratto, Yuri. 2010. <Los muros Megalíticos de Sacsayhuamán>. Revista de Historia y Medio ambiente, $\mathrm{N}^{\circ}$ 1, Santa Marta. < http://yurileveratto.com/articolo. php?Id=103> [Consulta: 25-08-2015].

Newstrom, John. 2011. Comportamiento Humano en el trabajo. México: Mc Graw Hill.

Urcola, Juan. 2010. Dirigir personas: fondo y formas. Madrid: ESIC.

Whetten, David y Cameron, Kim. 2011. Desarrollo de habilidades directivas. México: Prentice Hall.

Fecha de recepción: 13 de noviembre 2015

Fecha de aceptación: 07 de diciembre 2015

\section{Correspondencia}

Lorenzo Valderrama Plasencia

querubinp@hotmail.com 